\title{
Readout for intersatellite laser interferometry: Measuring low frequency phase fluctuations of HF signals with microradian precision
}

Oliver Gerberding, ${ }^{1, \text { a) }}$ Christian Diekmann, ${ }^{1, \text { b) }}$ Joachim Kullmann, ${ }^{1}$ Michael Tröbs, ${ }^{1}$ loury Bykov, ${ }^{1}$ Simon Barke, ${ }^{1}$ Nils Christopher Brause, ${ }^{1}$ Juan José Esteban Delgado, ${ }^{1}$ Thomas S. Schwarze, ${ }^{1}$ Jens Reiche, ${ }^{1}$ Karsten Danzmann, ${ }^{1}$ Torben Rasmussen, ${ }^{2}$ Torben Vendt Hansen, ${ }^{2}$ Anders Engaard, ${ }^{2}$ Søren Møller Pedersen, ${ }^{3}$ Oliver Jennrich,, ${ }^{4}$ Martin Suess, ${ }^{4}$ Zoran Sodnik, ${ }^{4}$ and Gerhard Heinzel ${ }^{1}$

1) Max Planck Institute for Gravitational Physics, and Institute for Gravitational Physics of the Leibniz Universität Hannover, Callinstrasse 38, 30167 Hannover, Germany

2) Axcon ApS, Diplomvej 381, DK-2800 Kgs. Lyngby, Denmark

${ }^{3)}$ DTU Space, National Space Institute, The Technical University of Denmark, Elektrovej 327, DK-2800 Kgs. Lyngby, Denmark

4) European Space Research and Technology Centre, European Space Agency, Keplerlaan 1, 2200 AG Noordwijk, The Netherlands

(Dated: 24 September 2018) 
Precision phase readout of optical beat note signals is one of the core techniques required for intersatellite laser interferometry. Future space based gravitational wave detectors like eLISA require such a readout over a wide range of $\mathrm{MHz}$ frequencies, due to orbit induced Doppler shifts, with a precision in the order of $\mu \mathrm{rad} / \sqrt{\mathrm{Hz}}$ at frequencies between $0.1 \mathrm{mHz}$ and $1 \mathrm{~Hz}$. In this paper, we present phase readout systems, so-called phasemeters, that are able to achieve such precisions and we discuss various means that have been employed to reduce noise in the analogue circuit domain and during digitisation. We also discuss the influence of some non-linear noise sources in the analogue domain of such phasemeters. And finally, we present the performance that was achieved during testing of the elegant breadboard model of the LISA phasemeter, that was developed in the scope of an ESA technology development activity.

Keywords: phase measurement, laser interferometry, instrumentation Submitted to Review of Scientific Instruments

\footnotetext{
a) contact@olivergerberding.com; Present address: National Institute of Standards and Technology, Gaithersburg, Maryland 20899, USA \& Joint Quantum Institute, University of Maryland, College Park, Maryland 20741, USA

b) Present address: SpaceTech GmbH, Seelbachstraße, 88090 Immenstaad am Bodensee, Germany
} 


\section{INTRODUCTION}

Inter satellite laser interferometers are studied for space based gravitational wave detectors, like LISA, eLISA ${ }^{1}$, and for satellite based geodesy missions, like GRACE Follow-On ${ }^{2}$. The orbit configurations of these missions lead to relative velocities of the spacecrafts, in the order of $10 \mathrm{~m} / \mathrm{s}$, that cause Doppler shifts of the laser beams that are transmitted between any two satellites. To accommodate these, a heterodyne detection scheme using readout systems with sufficiently large bandwidth, in the order of $25 \mathrm{MHz}$ (at a laser wavelength of $\lambda_{0}=1064 \mathrm{~nm}$ ) is the current baseline technique. Digital phase readout systems using all digital phase-locked loops (ADPLL), similar to GPS receivers, have been developed and utilised in various laboratories ${ }^{3-8}$.

The most critical aspect of these readout systems, also called phasemeters, is their phase noise performance over long time scales $(1000 \mathrm{~s})$, i.e. $\mathrm{mHz}$ Fourier frequencies. Especially the requirements for a future LISA like mission are very stringent. A noise floor below $2 \pi \mu \mathrm{rad} / \sqrt{\mathrm{Hz}}$ has to be achieved at frequencies between $5 \mathrm{mHz}$ and $1 \mathrm{~Hz}$, with a relaxed performance requirement below $5 \mathrm{mHz}$ (described by a noise shape function $\mathrm{NSF}(f)$ ). This corresponds roughly to a precision of $1 \mathrm{ppm}$ of the laser wavelength and translates to $1 \mathrm{pm} / \sqrt{\mathrm{Hz}}$ of effective optical path length change noise floor.

We have constructed a number of phasemeter prototypes that achieve such noise levels. This in particular includes a full breadboard model of the LISA phasemeter, developed in

the scope of an ESA technology development activity ${ }^{9,10}$, shown in Figure 1. Each of these prototypes utilises a distinct implementation of the analogue part of the phase readout chain, which is one of the most critical and challenging elements of the metrology. Due to our investigations we have gained detailed insight into the relevant effects and in this article we report our current best understanding of them, together with experimental results that demonstrate the desired performance levels.

\section{A. Phase readout chain overview}

To set the stage for our investigation we describe here shortly the core metrology chain for the phase readout in intersatellite interferometry, sketched in Figure 2. For readability we omit an introduction of the optical set-ups, which can be found in various related 


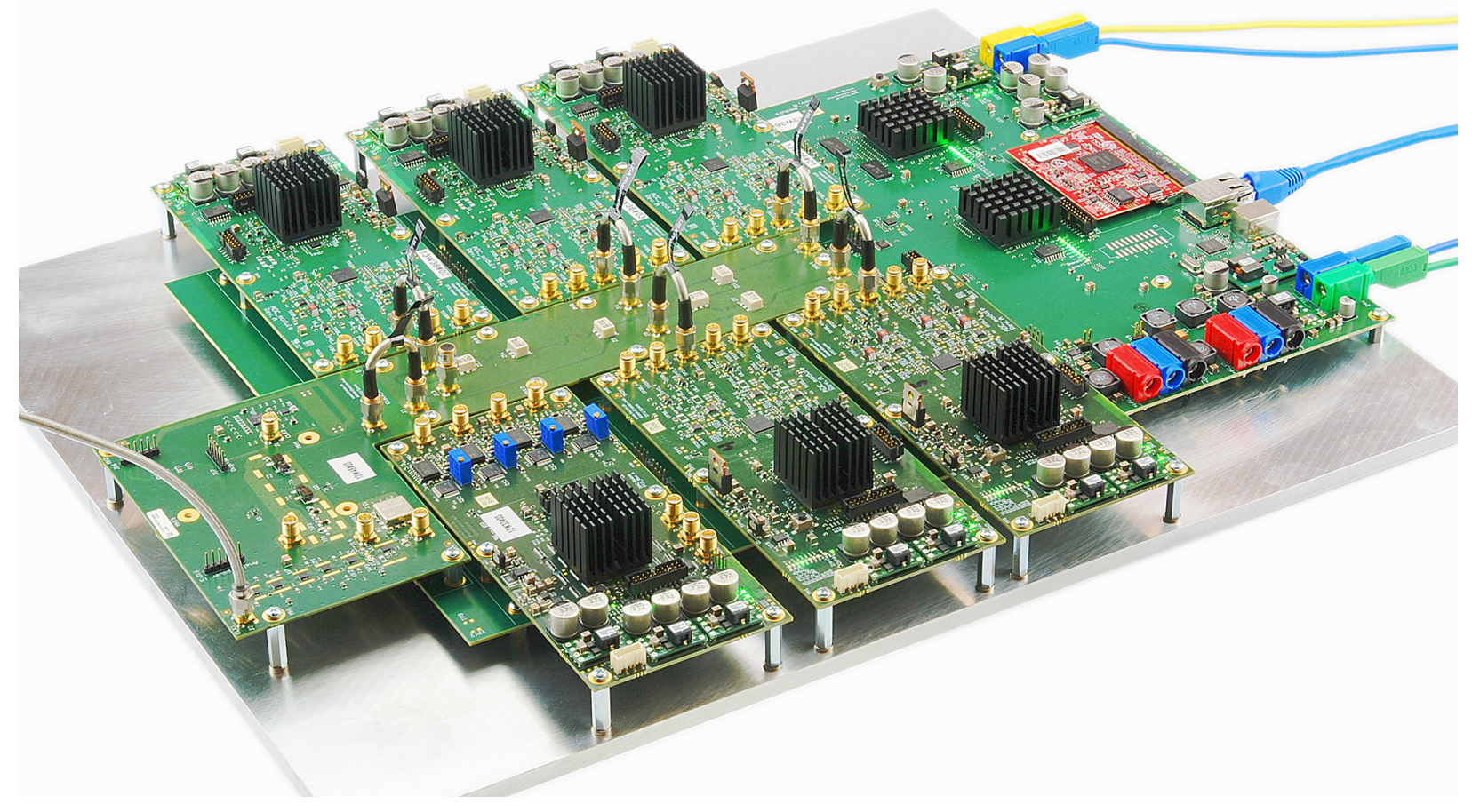

FIG. 1. Photograph of the assembled elegant breadboard model of the LISA phasemeter. The system consists of one main board, one clock module, one DAC module and up to five ADC modules $^{9}$. A total of 20 readout channels, 4 analogue control outputs, eight FPGAs and an ultrastable clock and pilot tone distribution are included.

publications $^{2,11,12}$.

The starting point of our description is an optical beat note with a frequency between about $1 \mathrm{MHz}$ and $25 \mathrm{MHz}$ (the exact numbers depend on the individual mission and its design) that is sent to a photo diode (PD). The frequency and phase of this beat note contain the desired information about length and attitude fluctuations (by differential wavefront sensing, DWS $)^{13}$ sensed by the interferometer. The PD is usually integrated with a transimpedance amplifier (TIA), that converts the photo current into a voltage, and, together, they form an active photo receiver $(\mathrm{PR})^{14}$. These devices are placed either directly in the interferometer or close by. Their outputs are usually single-ended analogue signals that are fed through coaxial cables to the phasemeter.

The phasemeter can, conceptually, be split into two parts: First, the signal conditioning in the analogue front-end (AFE) and the signal digitisation in the analogue-to-digital converter (ADC). Second, the deterministic digital signal processing (DSP) performed in 


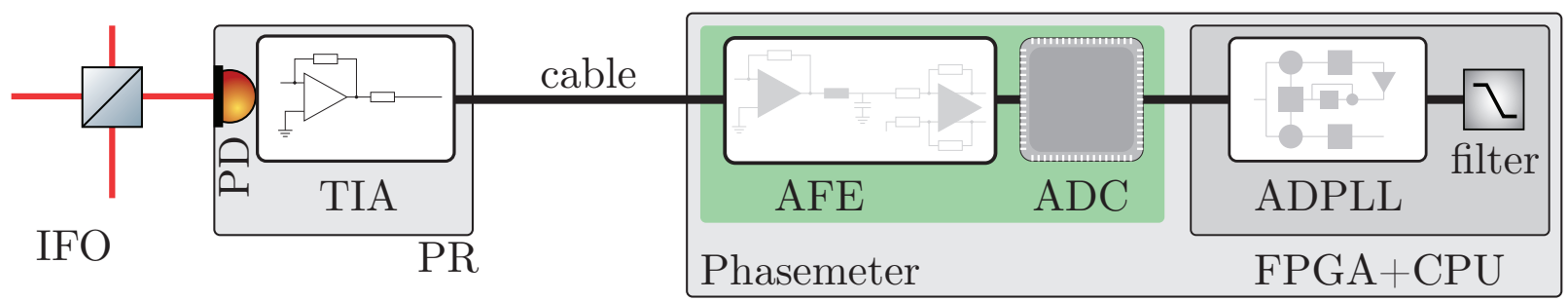

FIG. 2. Schematic overview of the phase readout chain. This article focuses on the elements marked green. The interferometer (IFO) signal is detected by a photo receiver (PR), which consists of a photo diode (PD) and a trans-impedance amplifier (TIA). The phasemeter receives and conditions the incoming signals in the analogue-front end (AFE) and then digitises it in the analogue-todigital converter (ADC). A combination of Field programmable gate arrays (FPGA)s and a central processing unit (CPU) extracts the signal phase via an all-digital phase-locked loop (ADPLL) and post processes the data for storage or further use.

FPGAs and CPUs that consists mainly of ADPLLs, appropriate filtering, decimation and signal combination.

The DSP units inside the phasemeter have already been described and investigated in detail $^{3,4}$ and will not be discussed further in this article. Based on this earlier work, we assume that the noise in the measurements shown below is not caused by artefacts in the DSP. However, for some of the later described, not-yet understood effects, an influence of the DSP can not be fully excluded at the current time.

The classification of the AFE as part of the phasemeter is arbitrary. All of the components can, in principle, also be part of the photo receiver or any other separate element in between the actual photo diode and the analogue-to-digital converter. The described analogue circuit effects are also present in the PR, however, they are often investigated separately, because PR are required in higher numbers, they have heritage from commercially available components and they operate in a different environment, close or on the optical benches, with, for example, better thermal stability. The concept of building independent PRs, that send single-ended signals to the phasemeter through an impedance matched cable has been widely used and is the basis for the discussions in this article. Alternatives that use differential signal distribution, digitisation directly on the photo receiver, and stand-alone analogue-front-ends that are separated physically from the digital components of the phasemeter are possible 
but were discarded so far, due to concerns regarding thermal design, power consumption and overall complexity. The strong influence of temperature fluctuations, described later in this article, might lead to a revision of these design principles for future studies and implementations.

\section{B. Article structure}

We begin our review with a conceptual discussion of the signal digitisation in the ADC (Section II). This is necessary to understand the requirements that drive the design of the AFE. The derived structure of the AFE is described next, together with a set of known noise sources (Section III). We then discuss non-linear effects in the AFE that can also limit the performance (Section IV).

The standard test procedure and a set of experiments using a combination of commercial hardware, and in-house produced components is presented to demonstrate successful implementations that were, however, limited in input frequency range. (Section V).

Next, we present the results of phase noise investigations of a breadboard model of the LISA phasemeter in Section VI, where realistic, LISA-like analogue input signals were used. This is accompanied with an overview of an active temperature stabilisation that was implemented to achieve, for the first time, full performance for signal frequencies of up to $25 \mathrm{MHz}$.

\section{ANALOGUE-TO-DIGITAL CONVERSION}

The interferometer signals need to be digitised with sufficient precision and rate. The two most critical sampling parameters are the sampling frequency $f_{s}$ and the ADC bit depth N.

\section{A. Truncation noise}

Quantisation noise induced by digitisation is often modelled as white additive noise, an assumption that, given the complex, noisy input signal characteristics expected in LISA like missions, is assumed to be appropriate ${ }^{15}$. The effective phase noise level due the quantisation noise depends not only on the sampling parameters ${ }^{3,7}$, but also strongly on the ratio between 
the signal peak amplitude $v_{\mathrm{s}}$ and the maximum ADC input voltage range $v_{\mathrm{ADC}}$. The effective phase noise $\widetilde{\varphi}_{\text {trunc }}$, due to truncation, can be written as ${ }^{4}$

$$
\widetilde{\varphi}_{\text {trunc }}=\frac{v_{\mathrm{ADC}}}{v_{\mathrm{s}}} \frac{2^{-N}}{\sqrt{3 f_{s}}} .
$$

Here, the largest possible signal before the occurrence of clipping and lowest phase noise corresponds to $v_{\mathrm{s}}=v_{\mathrm{ADC}} / 2$. Truncation, therefore, induces the need for proper signal gain conditioning in the AFE. The effective levels during digitisation should be as high as possible to minimise truncation noise influence, but they should also be small enough to avoid clipping. One should note, that the corresponding maximum signal calculation has to take additive input noise and additional tones into account.

\section{B. Aliasing}

During the sampling process any signals above the Nyquist frequency $\left(f_{s} / 2\right)$ are folded into the baseband. This does not only apply to coherent signals, but also to noise. This is a critical factor, since it means that additive noise sources, like, for example, photon shot noise, that have a bandwidth larger than $f_{s} / 2$, will accumulate after digitisation in the base band. However, these influences can be calculated and used to derive required suppressions of an anti-aliasing filter (AAF) in the AFE. A higher sampling rate reduces not only digitisation noise, but it also increases the band of frequencies that is not aliased into the signal bandwidth, simplifying the filter construction. Since faster sampling is usually accompanied by higher power consumption and other system design demands a trade-off has to be made. The response of the PR has to be taken into account as well, which will often already provide some level of suppression at higher frequencies.

\section{ADC sampling jitter and pilot tone}

During the digitisation the signal is sampled using the provided ADC clock. Any phase change in the distribution or application of this clock to the sampling process causes an effective timing jitter and a corresponding phase change in the measured signals. The phase error amplitude spectral density $\widetilde{\varphi}_{\widetilde{\tau}}\left(\left[\widetilde{\varphi}_{\widetilde{\tau}}\right]=\mathrm{rad} / \sqrt{\mathrm{Hz}}\right)$ scales with the input signal frequency and the timing jitter spectral density $\widetilde{\tau}([\widetilde{\tau}]=\mathrm{s} / \sqrt{\mathrm{Hz}})$ :

$$
\widetilde{\varphi}_{\widetilde{\tau}}=2 \pi f_{s} \cdot \widetilde{\tau}
$$


Experiments in the last decade have revealed that all suitable ADCs have an inherent $1 / f$ timing jitter noise that prevents phasemeters from directly achieving the desired performance levels. This noise is inherent to the ADCs themselves, and is present even with a perfect clock distribution.

However, the timing jitter can be accounted for by utilising a reference tone ${ }^{3}$, also called pilot tone, that is fed into all ADC channels. The phase deviations of this tone, whose frequency $f_{\mathrm{p}}$ is well known, can be converted into an effective measurement of the timing jitter. The corrected phase for each input channel $\varphi_{i, c}$ is given by the combination of the signal phase measurement $\varphi_{i, s}$ and the individual pilot tone measurement $\varphi_{i, p}$ :

$$
\varphi_{i, c}=\varphi_{i, s}-\varphi_{i, p} \frac{f_{i, s}}{f_{p}} .
$$

To implement such a correction scheme the AFE needs to be able to distribute the pilot tone, to add it to each individual signal and to deliver both signals after appropriate signal conditioning to the ADC, without spoiling the phase noise performance of either tone. The requirement for the pilot depends on the frequency ratio.

In a timing picture the pilot tone is the effective, jitter free (reduced) reference clock.

\section{ADC analogue parameters}

So far we have focused on the sampling parameters of ADCs. However, the analogue effects described in the following sections III and IV are also relevant for the ADCs. We have empirically tested candidate ADCs for compliance by directly performing pilot tone corrected phase noise measurements in relevant thermal environments, as described in Section V.

\section{ANALOGUE FRONT-END}

The signal conditioning for digitisation is done in the AFE. In addition to the above described functions the AFE also converts the single ended input signal into a differential signal with a desired gain and impedance to match the following ADC. The separate functions required of the AFE are listed in the following:

- Buffer: Receive PR signal from coaxial cable.

- Amplifier: Amplify signal with desired gain. 
- Anti-aliasing filter: Reduce unwanted signal components above the Nyquist frequency.

- Pilot tone distribution: Deliver the pilot tone to individual AFEs without introducing excess phase noise.

- Adder: Add the pilot tone to the PR signal before digitisation.

- Single-ended to differential converter: Prepare the signal for the ADC.

The critical effects in the AFE that contribute to phase noise are summarised below.

\section{A. Additive noise sources}

Any component in the AFE can contribute additive electronic noise. The induced corresponding phase noise is simply given by the ratio of the noise and the root-mean-square (RMS) signal amplitude at the given point in the measurement chain ${ }^{4}$. Hence, the most critical point for introducing noise is the one with the smallest signal. For LISA this is generally the first amplification in the trans-impedance amplifier of the photo receiver ${ }^{14}$. Signals sent to the phasemeter are assumed to be amplified to sufficiently high levels, such that fundamental noise from passive components, like Johnson noise from resistors, is sufficiently small.

Operational amplifiers and other active components used in the AFE can also introduce voltage noise in the $\mathrm{MHz}$ signal band. To achieve effective phase noise levels below $6 \mu \mathrm{rad} / \sqrt{\mathrm{Hz}}$ these contributions should be below $600 \mathrm{nV} / \sqrt{\mathrm{Hz}}$ for typical minimal signal RMS amplitudes of $100 \mathrm{mV}$. Commercially available low-noise operational amplifiers achieve noise levels on the order of a few $\mathrm{nV} / \sqrt{\mathrm{Hz}}$, which makes this contribution non-critical for the circuits used in the AFE, like inverting-amplifiers with moderate levels of gain (the noise coupling of operational amplifiers is gain dependent $\left.{ }^{16}\right)$.

\section{B. Phase noise}

Changes of the signal phase in the AFE directly spoil the measurement performance. Each component in the AFE can influence the phase of a transmitted signal and here we shortly describe two types of phase noise coupling that are relevant and occurred during our experiments. 


\section{Temperature coupling through finite phase response}

Components in the AFE have non-flat transfer functions $T(f)$. Any frequency dependent phase response, as, for example, induced by a simple signal delay, influences the phase propagation through the measurement chain. Constant, linear frequency dependent phase response is not critical for LISA, because the signal frequency changes are happening at frequencies below the measurements band $(<1 \mathrm{mHz})$. If significant frequency drifts occur within the measurement band, like expected for GRACE Follow-On ${ }^{2}$, the overall phase response either has to be sufficiently flat, or a correction in post-processing could be applied.

Even for a constant input frequency $f_{s}$ the phase response can cause significant phase noise if its value, $\arg \left(T\left(f_{s}\right)\right)$, is influenced by in-band fluctuations of temperature, for example due to the change of a resistance or capacitance. Flat transfer functions are in general less susceptible to these fluctuations, as the susceptibility to thermal variations depends on the absolute slope of the phase response. Accordingly, the phase of signals at higher frequencies is more susceptible to thermal changes in bandwidth limited circuits. A reduction of temperature fluctuations is one way to mitigate the induced phase noise (as we present in Section VI), but limitations in size, power consumption and cost of the apparatus limit this approach. Performing detailed analysis of each components susceptibility to temperature changes is required to reduce the coupling itself. Choice of more stable components and thermal compensation are some of the methods that can be applied. A rule of thumb that we applied during our investigations is to use components with as large a bandwidth as possible. This approximates flat transfer functions responses that minimise the coupling to thermal effects.

Due to this coupling the most critical component in the AFE is the anti-aliasing filter. It requires a large suppression above the Nyquist frequency to ensure shot-noise limited performance (see Section II), which strongly limits its bandwidth and phase response flatness, and it has to have sufficiently low coupling of thermal noise into phase noise. Our investigations presented here do not include a detailed analysis of, nor a design for, the AAF. Based on

our later presented results it is assumed, however, that integrating the AAF into the PRs might be beneficial, due to the better thermal stability. 


\section{Flicker phase noise}

Another source of phase noise is flicker noise, that is, for example, induced by a $1 / f$ noise at DC in amplifiers that effectively modulates the carrier phase ${ }^{17}$. In our experiments we have avoided using components that contribute any such excess noise, by performing empirical tests. The investigation of such effects is a separate topic that goes beyond the work presented here and we found no essential components that are limited by them. Frequency mixers are a prominent example of components limited by such noise and we have observed it in earlier studies ${ }^{4}$.

\section{Pilot tone distribution}

The use of a pilot tone requires it to be distributed to each analogue measurement channel. This involves impedance matched signal splitting, signal routing, and, as discussed later in Section IV, isolation between channels. The phase noise analysis done for the AFE also has to be applied to the pilot tone distribution (PTD), being, however, slightly simplified because the pilot has a single, fixed frequency.

\section{NON-LINEAR EFFECTS}

The phase measurement effects described above are critical for understanding and designing phasemeter systems. So far we have described the influence of processes that either cause an additive noise or a phase noise of the coherent signal. Both of these noise types cause an effective phase noise (additive noise is converted into phase noise in the mixer in the ADPLL), whose underlying processes are linear with respect to the noise amplitude within the bandwidth of the ADPLL. But, other types of interactions can also limit the achievable performance. The one we consider here is the presence of additional, parasitic tones at the signal frequency or close by. The ADPLL does not distinguish between the actual signal tone and the parasitic one and will act as if a single disturbed tone is present (parasitic tones outside the ADPLL bandwidth are rejected).

A simple way to determine the combined, disturbed tone is to describe both tones as phasors in the complex plane at the readout frequency. The two phasors, or 2-dimensional vectors, each defined by an amplitude and a phase, are added, resulting in the effective 

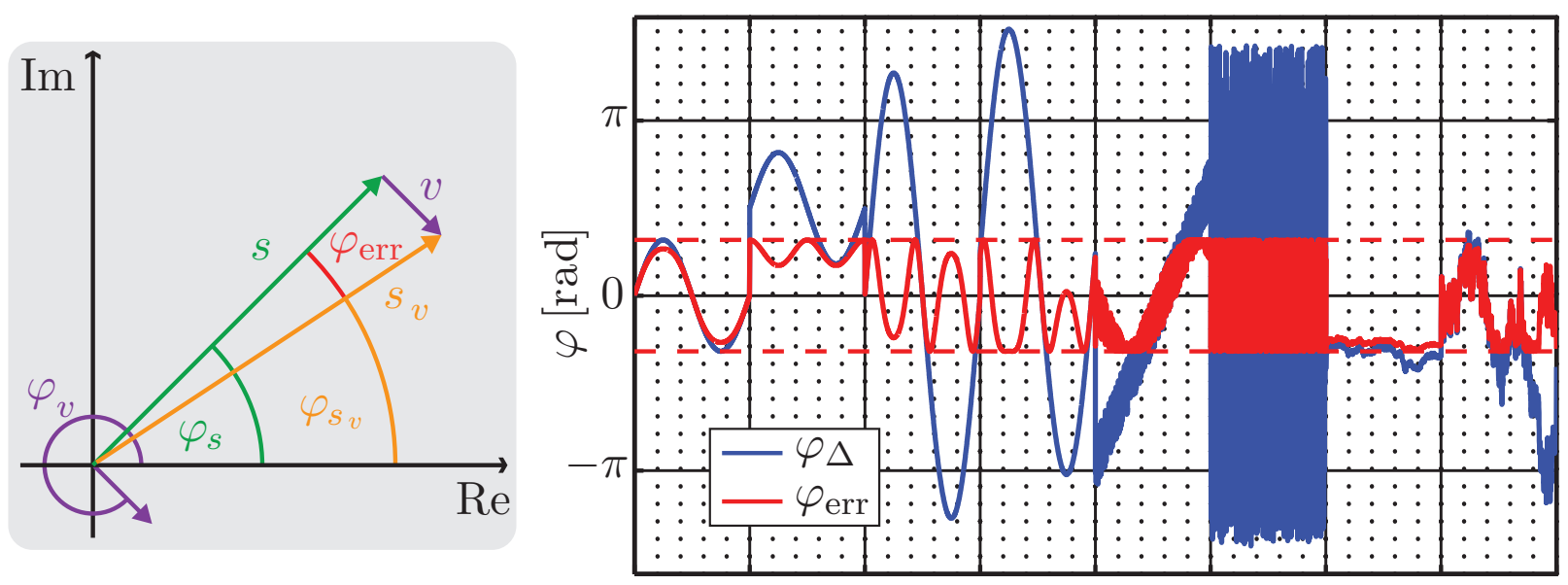

FIG. 3. Illustration of small vector noise coupling. The left side shows a sketch of the vector addition responsible for the non-linear noise coupling in case of small vector noise. The right side shows a simulated time series of a small vector phase modulation for coherent modulations with different DC offsets and amplitudes and for white noise and $1 / f$ noise.

phasor, with a corresponding phase and amplitude. The difference between the effective phase and the phase of the signal tone is the phase error. The vector addition causes the coupling into the measurement of phase or amplitude changes of the parasitic tone relative to the signal tone to be highly non-linear. A parasitic tone that has a constant phase and amplitude ratio relative to the signal, will only result in a DC phase error, which is anyhow neglected for longitudinal displacement measurements (not for DWS though).

The parasitic signals become more relevant when they have non-negligible dynamics relative to the signal tone. Such unwanted, parasitic tones are made as small as possible in comparison to the signal in the system design. Their effect is illustrated in Figure 3 . In this case, a small phasor, or vector, $\vec{v}_{\mathrm{v}}$ is added to the larger, dominating signal tone $\vec{v}_{\mathrm{s}}$. The phase noise caused in such a situation is often referred to as small-vector noise. The complex coupling of relative phase fluctuations $\left(\varphi_{\Delta}=\varphi_{\mathrm{s}}-\varphi_{\mathrm{v}}\right)$ into phase error $\varphi_{\text {err }}$ can be approximated for the small parasitic vector to

$$
\varphi_{\mathrm{err}}=\frac{v_{\mathrm{v}}}{v_{\mathrm{s}}} \sin \left(\varphi_{\mathrm{s}}-\varphi_{\mathrm{v}}\right)
$$

Such non-linear noise couplings are well known in the gravitational wave community. A small vector noise has been observed and suppressed in LISA Pathfinder ${ }^{18}$, and stray light and ghost beams, which are present in many laser interferometers, cause a very similar effect 
as well.

In case of small relative phase dynamics $\varphi_{\Delta}$, and DC phases close to the linear regimes of Equation 4, the induced phase noise is linear to the dynamics, and its amplitude is attenuated by the ratio $\frac{v_{\mathrm{v}}}{v_{\mathrm{s}}}$. In the more general case of large dynamics and arbitrary phase relations, no simple method exists to predict the phase error from the relative dynamics. Some examples for this coupling are shown in simulated time series on the right of Figure 3. Large low frequency changes can be unconverted to higher frequency errors, due to the non-linear coupling. An important feature of this noise coupling is, that the induced

phase error has a maximum amplitude, which is also defined by the ratio $\frac{v_{\mathrm{v}}}{v_{\mathrm{s}}}$. This leads to a characteristic and often diagnostically useful time-domain signature of a constant width envelope. Small vector noise driven by low-frequency random processes with a $1 / f$ spectrum, like, for example, temperature fluctuations, often show a characteristic phase noise spectrum. Such a spectrum flattens out to lower frequencies (due to the maximum error amplitude) and a characteristic noise shoulder is observed at the high frequency end of the generated noise bandwidth. If the readout bandwidth of the phasemeter is low in comparison to the generated noise, the drop-off to higher frequencies might not be observed. In this case a small vector noise can be misinterpreted as white readout noise and the underlying non-linear coupling makes eventual noise hunting more complex.

\section{A. Cross talk}

The most relevant parasitic tones in a multi channel phasemeter are cross talk components from other channels operating at the same, or nearby frequencies. The phase of these tones can be totally unrelated, potentially causing a very dynamic behaviour of $\varphi_{\Delta}$. This is, for example, the case for the two test mass and reference interferometers on the two optical benches in a LISA satellite ${ }^{19}$. The parasitic voltage in the case of cross talk can be modelled by the product of the amplitude of the unrelated signal $v_{\mathbf{u}}$ with a cross talk coupling factor $C$. Assuming a coupling as described in Equation 4, equal amplitudes for all input signals $\left(v_{\mathrm{u}}=v_{\mathrm{s}}\right)$, and a performance requirement of better than $2 \pi \mu \mathrm{rad}$, a crosstalk suppression in the order of $C=-105 \mathrm{~dB}$ is necessary. This level is determined from a simple maximum error calculation. Better estimates can be made by applying full models of the expected phase dynamics and using them to make a prediction of the behaviour of $\varphi_{\Delta}$. 
Cross talk can occur at every stage of the analogue signal processing, including, prominently, the $\mathrm{PR}^{20}$. Effects like electro-magnetic interference, power supply or ground stability can limit the achievable cross talk suppression between individual channels. Mitigating these effects requires detailed analysis and design of circuits, layouts, PCB stacks, component placements, electro-magnetic shielding and more. These investigations exceed the scope of this article. However, we will briefly discuss the two most critical points in the AFE of our phasemeter systems where the achievable limit of cross talk suppression is determined by component choice and simple, schematic level circuit design.

Multi channel ADCs have inherent cross talk, often with a coupling of about $-90 \mathrm{~dB}$, which is documented in their data sheets. Hence, using multi-channel ADCs for the readout of independent IFOs should be avoided to mitigate this problem. On the other hand, using them to read out the four signals of a quadrant photo diode is suitable, because these signals are already mixed due to the cross talk in the PR, and they are very similar. They are dominated by the DWS signal, keeping the value of $\varphi_{\Delta}$ small and even well measured.

The pilot tone distribution is a direct link that connects all input channels. Sufficient care has to be applied to ensure that no significant portion of the signals is transmitted back through the PTD to other signals. The adder components in the AFE are critical, they should provide sufficient initial suppression of backwards reflected signals. Additional isolating components (buffers, filters, isolators) can also be introduced into the PTD, as long as they don't contribute excess phase noise.

\section{B. Analogue reflections}

Small vector noise can also be caused by a tone that is contaminated with a small delayed version of itself. This can occur if signal reflections are present, for example due to impedance mismatches. Figure 4 shows a simple model of such an effect. A signal is fed from the photo diode (the source with impedance $Z_{\mathrm{s}}$ ) through a cable (impedance $Z_{\mathrm{c}}$ and length $l_{\mathrm{c}}$ ) to the phasemeter (the load with impedance $Z_{1}$ ). The impedance mismatches give rise to two reflection coefficients, here denoted as $r_{\text {in }}$ and $r_{\text {out }}$. For small reflectivities the amplitude of the first delayed signal $v_{s}^{\prime}$ reaching the load (we discard multiple reflections) is attenuated by $r_{\text {in }} \cdot r_{\text {out }}$. The amplitude of the actual signal $v_{s}$ is, however, almost undisturbed. The phase of $v_{s}^{\prime}$ relative to $v_{s}$ is determined by its dynamics and by twice the delay $\tau_{\mathrm{c}}$, which is given 
by the cable length and the effective signal speed, in typical coaxial cables $\tau_{\mathrm{c}} \approx l_{\mathrm{c}} /(2 / 3 c)$. Using these relations and Equation 4 we can estimate the induced phase noise by such an analogue reflection:

$$
\varphi_{\mathrm{err}} \approx r_{\mathrm{in}} r_{\mathrm{out}} \sin \left(2 \frac{d \varphi_{\mathrm{s}}}{d t} \tau\right) \approx r_{\mathrm{in}} r_{\mathrm{out}} \sin \left(3 \frac{d \varphi_{\mathrm{s}}}{d t} \frac{l_{\mathrm{c}}}{c}\right)
$$

The expected signal dynamics for inter-satellite laser interferometry are quite large (they are dominated by laser frequency noise the level of which depends on the applied stabilisation scheme). Assuming the laser frequency noise $\tilde{f}$ is still small in comparison to the delay we can simplify the coupling,

$$
\widetilde{\varphi}_{\mathrm{err}}(f) \approx \frac{3 r_{\mathrm{in}} r_{\mathrm{out}} \tilde{f}(f) l_{\mathrm{c}}}{c} .
$$

Even for large levels of in-band laser frequency noise $(3000 \mathrm{~Hz} / \sqrt{\mathrm{Hz}})$ and cable lengths on the order of $2 \mathrm{~m}$ this coupling only becomes relevant for large reflection coefficients of more than $10 \%$. However, if laser frequency noise levels exceed the linear range, even at much lower frequencies, effective noise levels can be upconverted to in-band Fourier frequencies, due to the non-linear coupling. Our presented model is largely simplified and aims to give insight into a possible explanation for an unexpected noise floor that we have observed in the later described measurements, which was found to be somewhat influenced by signal dynamics, cable lengths and impedance matching. Influences of our more complex signal networks, including the pilot tone distribution, and of frequency dependent impedance values have not yet been accounted for and might need to be analysed in future studies.

\section{PERFORMANCE INVESTIGATIONS USING PHASEMETER PROTOTYPES}

A standard technique for investigating the noise of phase readout systems is the so-called null or zero measurement. A single signal is split into two or more readout channels and the differences in the measurement phases are used to investigate the noise floor. Figure 5 shows this principle for a two channel measurement. Throughout our investigation we performed multi-channel measurements and we plot the noise for each channel $\widetilde{\varphi}_{i}$ by computing the difference between its phase and the measured phase average:

$$
\widetilde{\varphi}_{i}=\varphi_{i, s}-\frac{1}{N} \sum_{k=1}^{N} \varphi_{k, s} .
$$




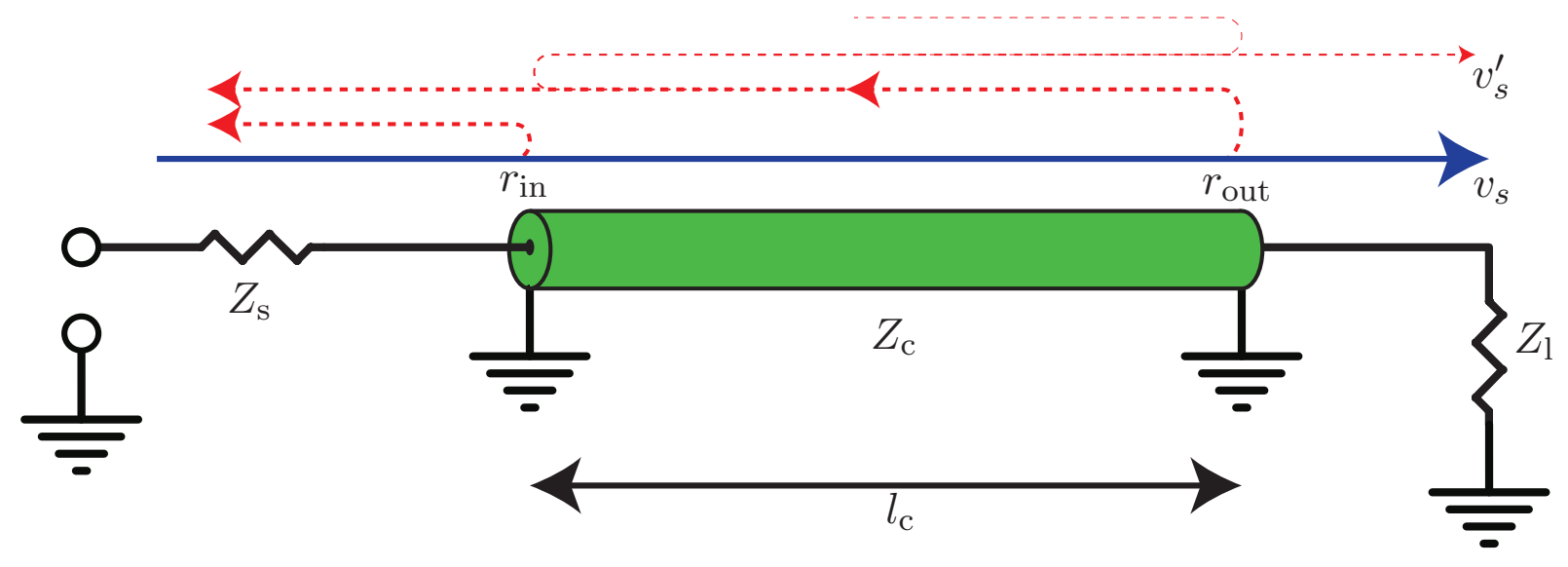

FIG. 4. Sketch for signal reflections occurring in the signal distribution from, for example, the photo diode to the analogue-front end. For small reflection coefficients $r_{x}$ a dominating parasitic signal $v_{s}^{\prime}$ is delayed by twice the cable length $l_{c}$ and contaminates the actual signal $v_{s}$.

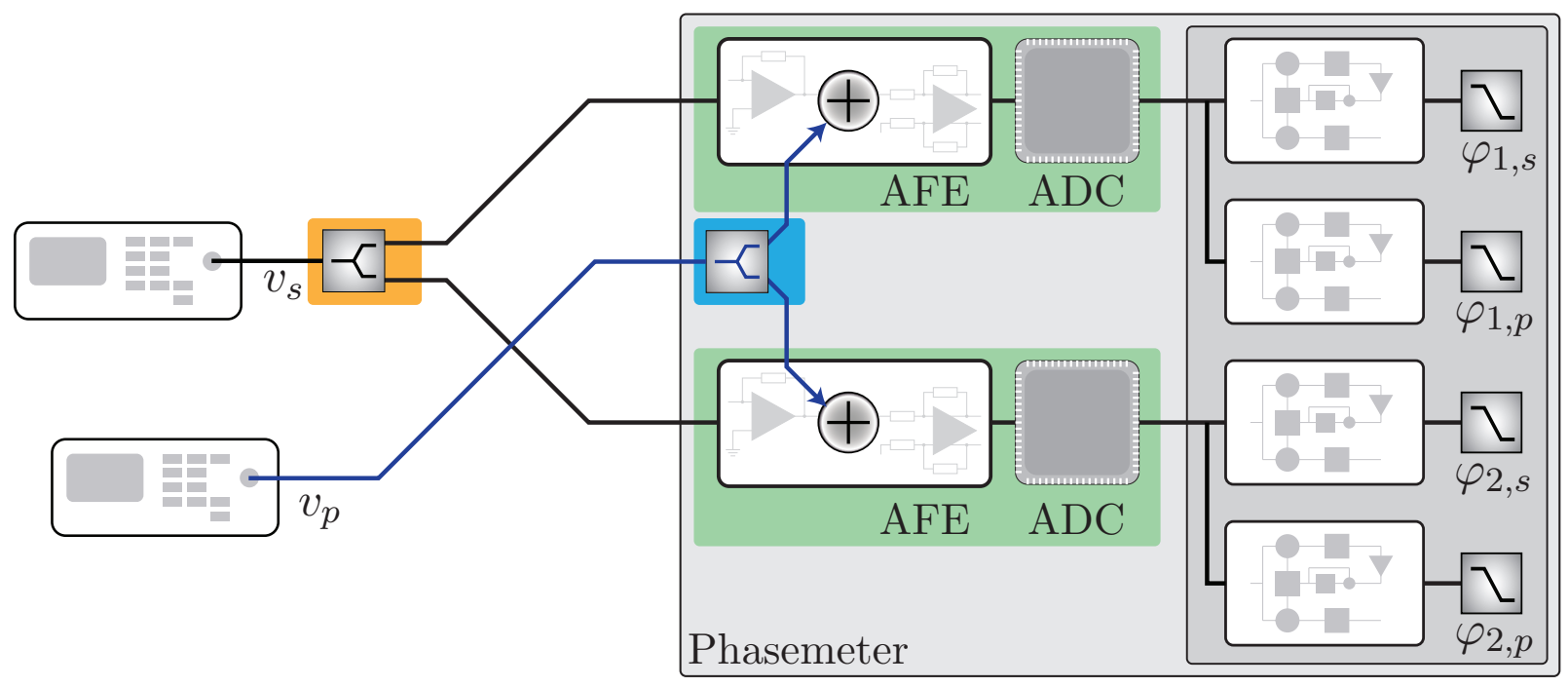

FIG. 5. Sketch of a null or zero measurement with two channels. The signal is split in a signal splitter (marked in orange) and fed into two readout channels. A pilot tone is also fed to the phasemeter, split and distributed via the PTD (marked blue) and added. Two all-digital phaselocked loops are used to read out the phase of the signal and the pilot tone for each channel.

This type of measurement is easily implemented and well suited to hunt critical noise sources. But, the subtraction of almost equal signals makes this scheme insensitive to common nonlinear effects ${ }^{4}$. Cross talk influence, for example, can not be measured well, since all channels already measure the exact same signal. Noise due to thermal fluctuations is, if it is common 
between all channels, also not detectable by such measurements. Common additive noise can, in the linear operating range of the ADPLL, not be detected either. The noise floor due to uncorrelated additive noise depends on the number of compared channels, as evident from Equation 7.

An important first result of our investigations is that some commercially available ADC circuits, using amplifiers for ADC signal conditioning, are capable of achieving the desired phase noise performance levels even in rather uncontrolled thermal environments. These systems only require the addition of a pilot tone to the input signals to enable the necessary timing jitter correction. We used this fact and combined an FPGA with a suitable ADC card (FMC107 from 4DSP ${ }^{21}$ ) to evaluate the feasibility and performance of different pilot tone distribution and adding schemes. For these measurement we used a rather stable input signal, produced by a commercial signal generator, and split and distributed it via a thermally isolated, resistive power splitter (also referred to as resistive tee). Thereby, we ensured that no significant phase noise was introduced by the signal distribution.

\section{A. Active front-end}

We tested two schemes using an active pilot tone adder. High-bandwidth operation amplifiers were used in simple inverting adder circuits (also referred to as summing amplifiers) to combine the signals and the pilot tone. The PTD was implemented in two ways, once using a resistive $50 \Omega$ matched splitter, and once using commercial, transformer based power splitter (also referred to as hybrid coupler) with a flat amplitude response in the signal bandwidth. Both options were integrated, together with the adders, on a separate circuit board, which was connected to the signal source and to the ADC card using coaxial cables. Sketches of the implemented schematics are shown in Figure 6, a) and b). A passive thermal isolation of the components was added by wrapping them in bubble wrap foil.

The achieved phase noise performance levels are shown in Figure 7 for the resistive power splitter set-up, the levels are also representative for the once achieved with the set-up using transformer based power splitters. The dashed lines represent the spectral density of the differences of the phase errors for the uncorrected signals. The straight lines show the same signals corrected for pilot tone jitter. Interestingly, one of the channels $(\mathrm{C})$ shows an increased phase noise before correction. This is, however, sufficiently suppressed afterwards. 

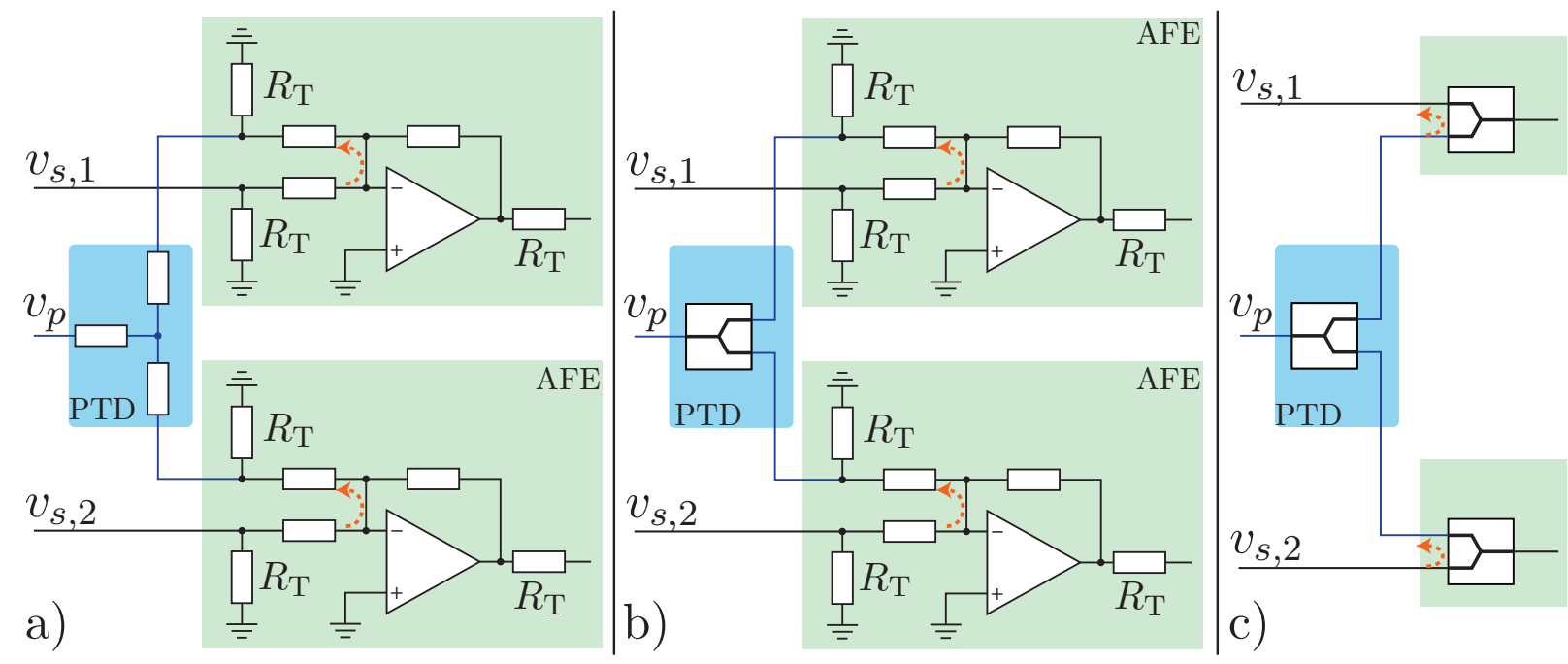

FIG. 6. Sketch of the schematics for the tested pilot tone addition schemes, each one is shown for two channels. a) Active pilot addition and distribution via resistive power splitter. b) Active pilot addition and distribution via transformer based power splitter and addition via inverting adder circuits. c) Passive pilot addition and distribution via transformer based power splitters. The orange arrows indicate signal leakage into the PTD that leads to cross talk.

Both schemes were able to achieve the desired performance levels. This demonstrates that the operational amplifier based adding circuits, as well as the pilot tone distribution via resistive network or commercial power splitters are suitable to perform at the desired noise levels for the specific set of frequencies used in each measurement.

In terms of suppression of crosstalk via the PTD the power splitter implementation is considered favourable. A cross talk signal introduced into the PT chain in one of the adders is attenuated by the isolation of the power splitters (typically $30 \mathrm{~dB}$ ) before it can reach another signal channel. A summing amplifier circuit provides a strong isolation between inputs, due to the virtual ground at the inverting input, which gives a strong initial cross talk suppression. However, a reduction of the nominal cross talk coupling via the PT distribution to less than $105 \mathrm{~dB}$ requires additional components.

\section{B. Passive front-end}

Based on the positive results with the passive pilot tone distribution an effort was started to test a fully passive PTD and adder that uses two types of commercial, transformer based 


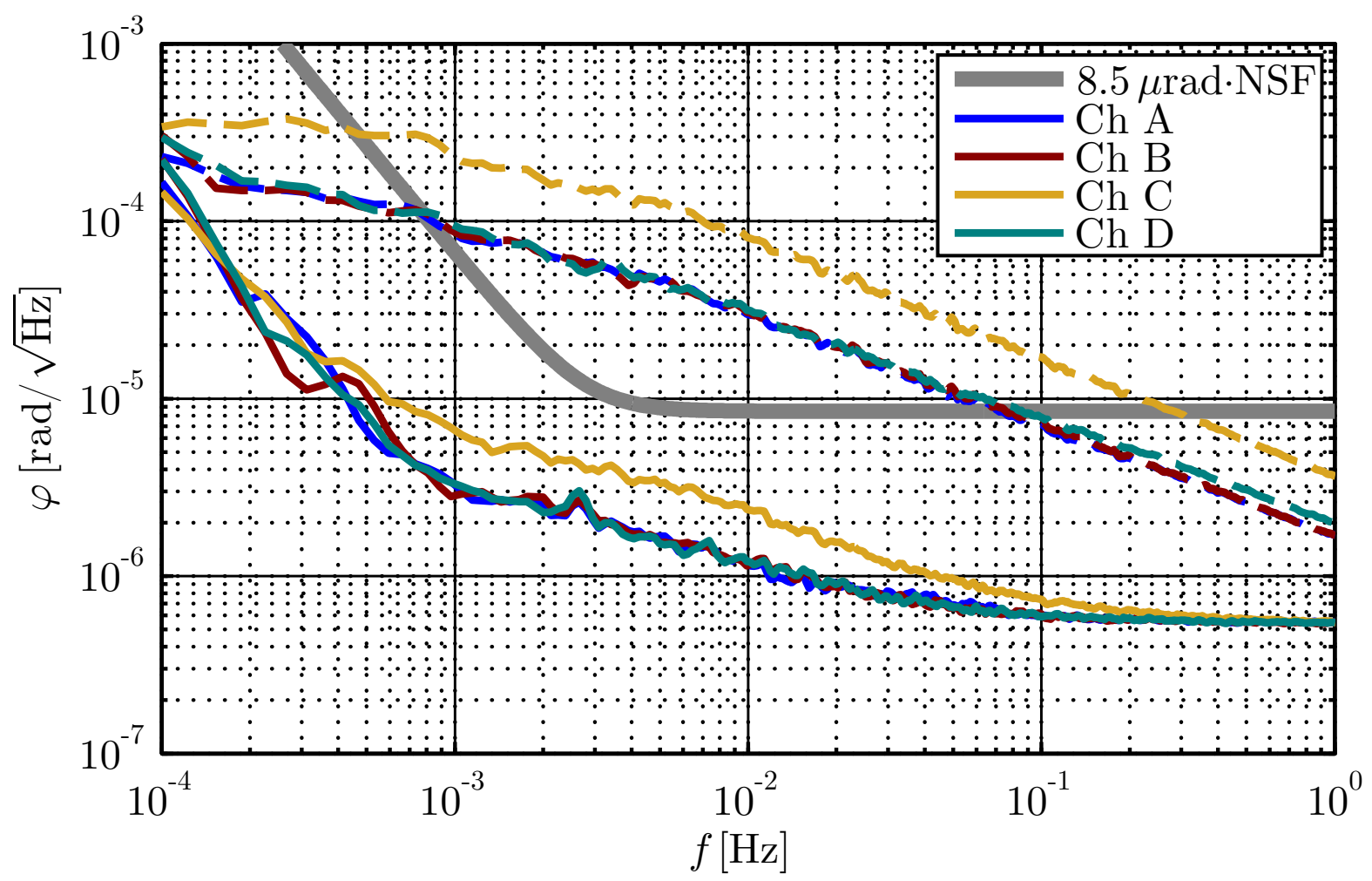

FIG. 7. Performance of active pilot tone adder and resistive distribution. The signal frequency was at $11 \mathrm{MHz}$, the pilot tone at $33 \mathrm{MHz}$. Excess noise in $\mathrm{C}$ is not understood. The dashed lines show the performance without jitter correction.

power splitters. The benefit of such an implementation is that it consumes no power and thus generates very little heat, which simplifies any thermal isolation schemes.

We have constructed AFEs with two different power splitters, one with a plastic housing (ADP-2-1, Mini-Circuits) and one in a metal housing (PSC-2-1, Mini-Circuits). Separate circuit boards containing the PT adder were, again, placed in passive thermal isolation and connected to the ADC card using impedance matched coaxial cables. A sketch of the schematics is shown in c) in Figure 6. We investigated the phase noise performance and found that both implementations were also able to achieve the desired levels for some specific set of signal frequencies. Figure 8 shows the performance for the set-up using the PSC-2-1, which is also representative for the performance achieved with the ADP-2-1.

We investigated the described schemes only with the given temperature stability and for a small set of frequencies, because the prototypes were aimed at single frequency operation. Choosing smaller (below $\approx 10 \mathrm{MHz}$ ) or higher (above $\approx 20 \mathrm{MHz}$ ) input frequencies did, 


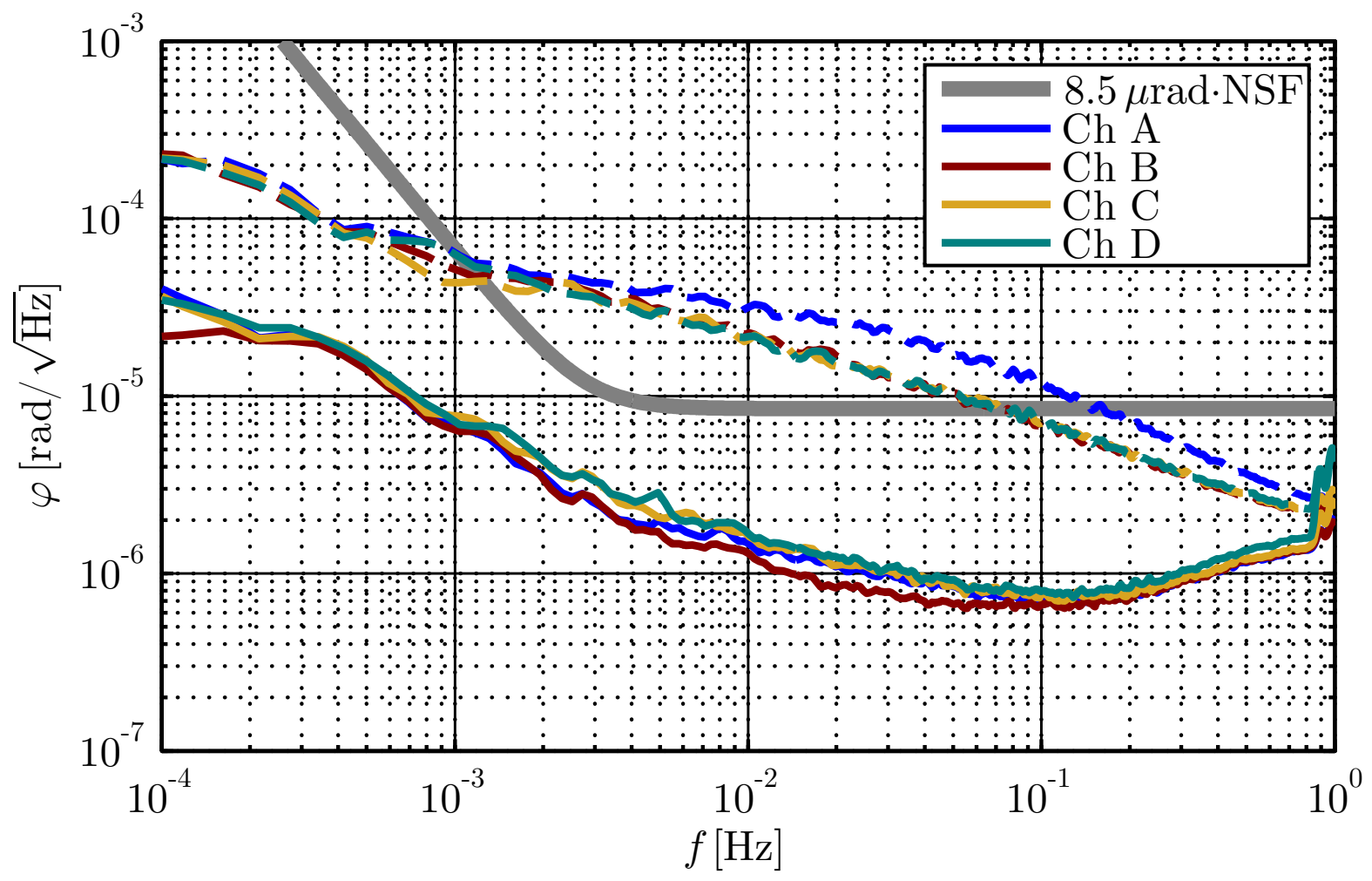

FIG. 8. Performance of passive pilot tone adder and distribution with PSC-2-1. The signal frequency was at $15 \mathrm{MHz}$, the pilot tone at $35 \mathrm{MHz}$. The dashed lines show the performance without jitter correction.

however, indicate the presence of excess noise. This was expected due to the bandpass characteristic of the transformer based power splitter. Their non-flat transfer function at lower and higher frequencies increases the coupling of temperature fluctuations into phase. It is currently not known, if this could have been reduced by further improved thermal stability to acceptable levels.

\section{16 channel prototype}

Based on the availability of a suitable PT adder scheme we designed an integrated phasemeter prototype that was aimed at achieving LISA performance levels at a single frequency with 16 channels. In terms of cross talk we decided to only aim for a suppression level of $60 \mathrm{~dB}$. This was limited by the cross talk induced on the utilised commercial ADC card (FMC116 from 4DSP ${ }^{22}$ ). 

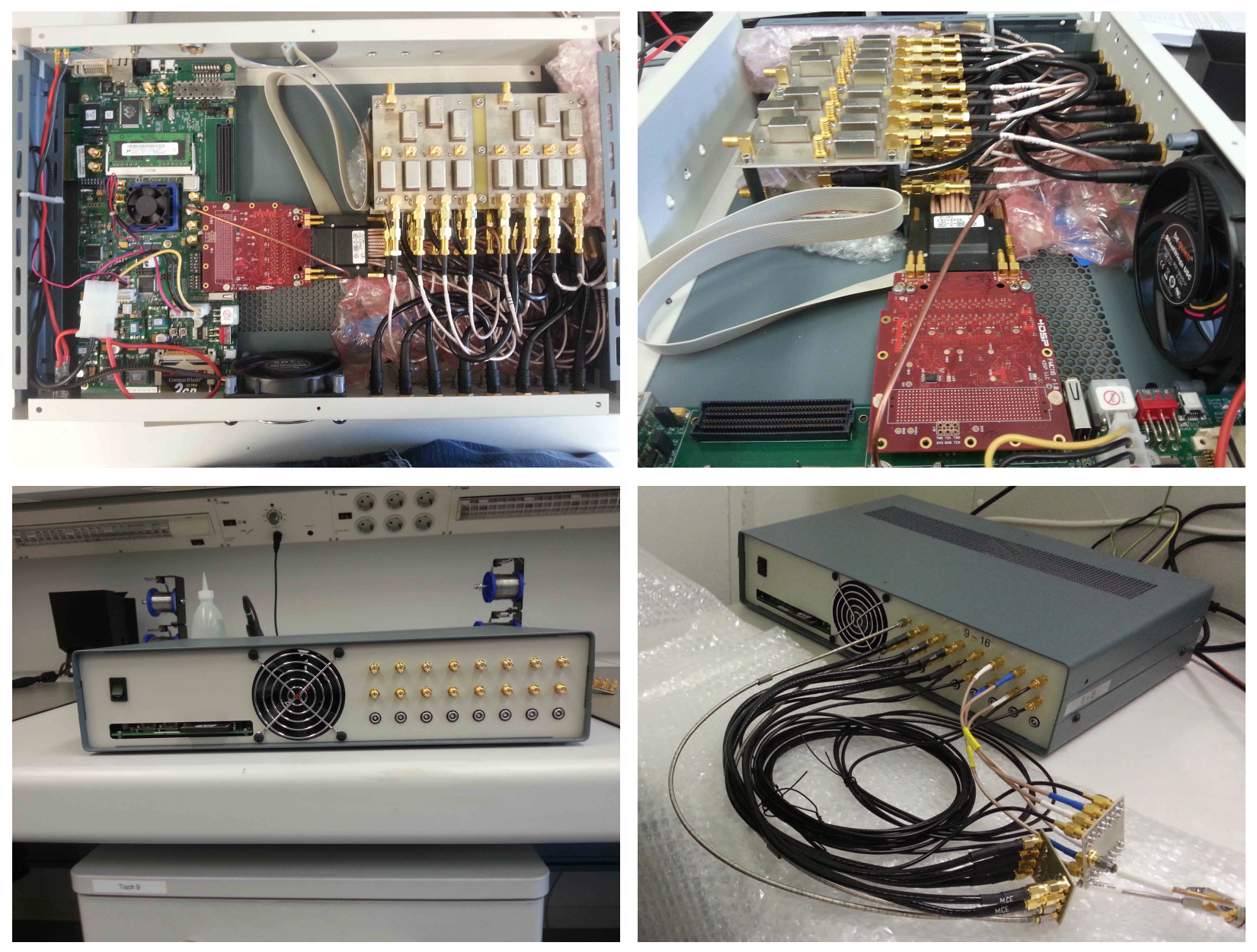

FIG. 9. Photographs of the 16 channel phasemeter built using commercially available components in combination with a passive pilot tone distribution and adder circuit.

We chose the implementation relying only on the transformer based power splitter PSC2-1. Two circuit boards with splitter networks were manufactured and mounted into a common housing with the FPGA board and ADC card, as shown in Figure 9.

Using a signal distribution with one two-way resistive splitter, and two eight-way resistive splitters we fed the same input signal into all 16 channels. Again, we were able to achieve $\mu$ rad performance with sufficient thermal isolation of the splitters and cables. However, the white noise levels in each channel showed varying levels, as shown in Figure 10, which were found to correspond to the cable length used for distributing the signals from the splitters to the phasemeter. Using cables with matched lengths allowed us to reduce this noise floor.

At this point we were not able to fully identify this noise coupling. One speculation is that the white noise is actually caused by a small vector noise present in all channels, which 


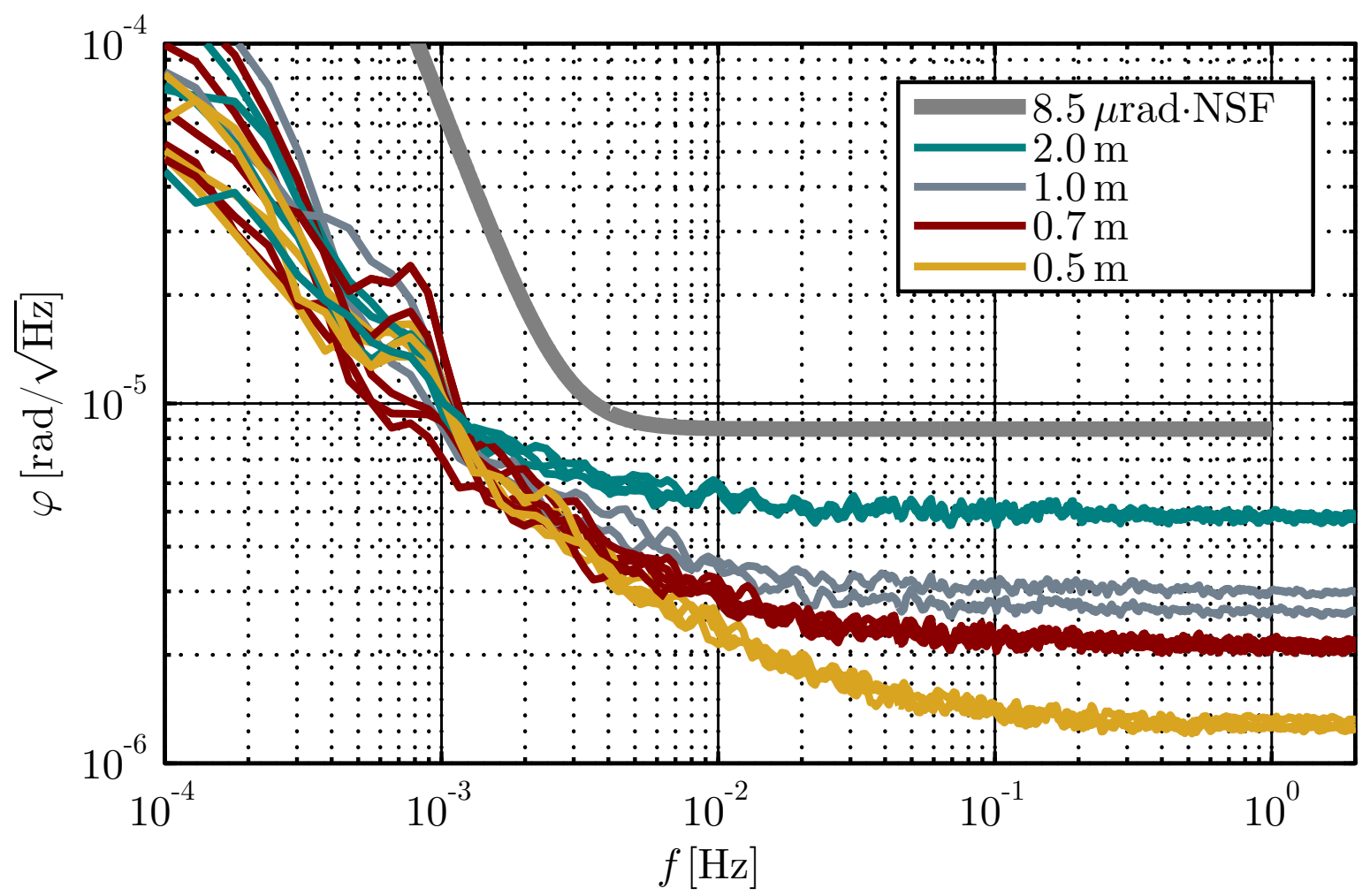

FIG. 10. Performance measurement using the 16 channel phasemeter. The signal frequency was at $13 \mathrm{MHz}$, the pilot tone at $35.3 \mathrm{MHz}$. The influence of the different cable lengths (colour coded) on the achieved noise floor is clearly visible.

might be caused by impedance mismatches in the signal or PT distribution, by the cross talk between the channels, or a combination of these effects, with the phase of the parasitic tone relative to the actual signal driven by the dynamics of the signal itself. The different cable lengths change the effective coupling in each channel, ultimately leading to the observed white noise floor given by the maximum amplitudes of the small vector noise. A similar effect is observed in the experiment presented in the next section.

\section{LISA PHASEMETER ELEGANT BREADBOARD}

In the scope of the LISA metrology system technology development project we built and tested a full breadboard of the LISA phasemeter ${ }^{9,10}$ (see Figure 1). For this system we implemented an active AFE using operation amplifiers and a PTD using transformer based power splitters on the clock module and impedance matched distribution on the ADC cards. 

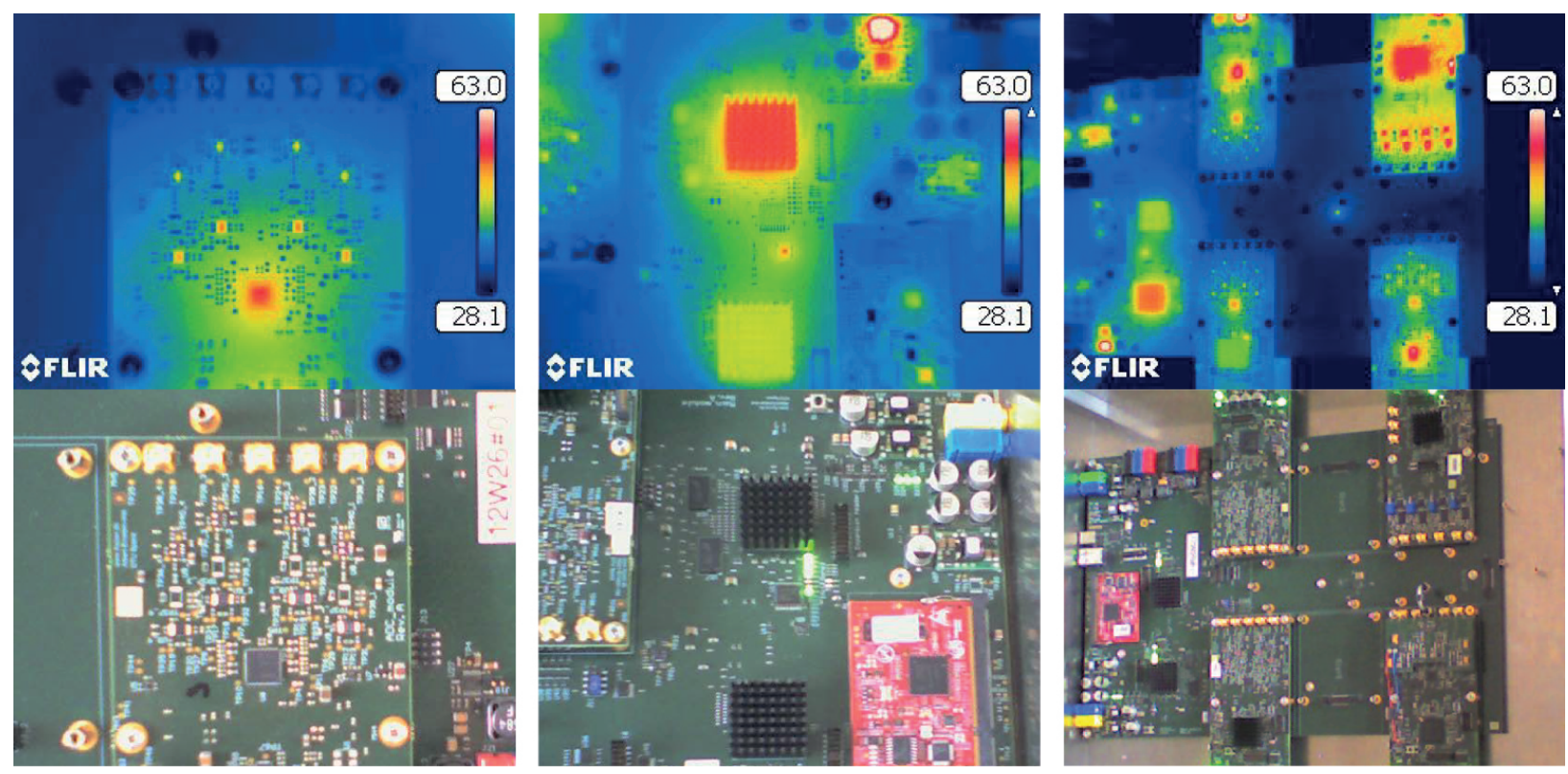

FIG. 11. Shown are thermal and corresponding optical pictures of the elegant breadboard model, taken about 20 minutes after start-up in air. The left image shows a close-up of the AFE, showing the heat dissipated by the ADC, which causes a thermal gradient through the AFE. The middle image shows the bridge FPGA and the FFT FPGA ${ }^{9}$, both with passive coolers attached to them, and one of the systems power supplies in the upper right corner. The FFT FPGA has about twice the amount of logic gates and, therefore, a higher power consumption. The right image shows the overall systems with three ADC modules and one DAC module. The DAC card generates significantly more heat. The FPGA temperature also varies strongly on the ADC modules, depending on whether they have a passive cooler mounted or not.

To determine the performance of our system we conducted a comprehensive measurement campaign using LISA like analogue input signals, which were generated by a dedicated signal simulator. These signals included a signal tone that had the dynamics of the expected laser frequency noise (the same conservative spectrum was used in our DSP study ${ }^{4}$ ) and a constant frequency drift. The synthesised signal also included two smaller side bands at $\pm 1 \mathrm{MHz}^{23}$, a weak 1.25 MHz modulation of pseudo-random noise ${ }^{24,25}$, and a white noise level with variable signal-to-noise ratio. We performed measurements at various signal frequencies, covering the range from $7 \mathrm{MHz}$ up to $25 \mathrm{MHz}$. The input signal in each case was split using an 8-way resistive splitter and short cables of equal lengths. These were connected to two ADC cards, allowing us to investigate the noise between channels on a single card, and between 


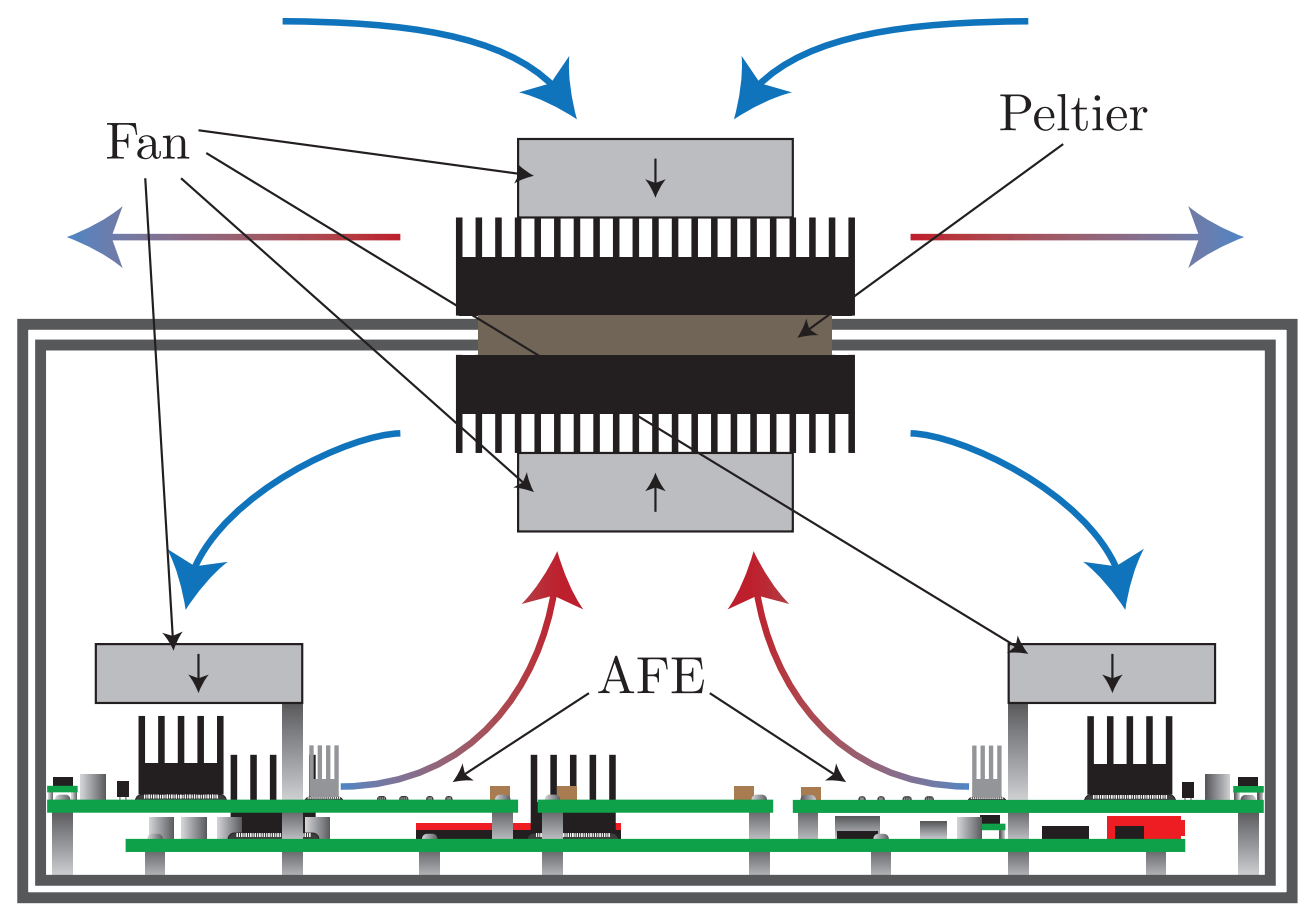

FIG. 12. Sketch of the active temperature stabilisation scheme. The temperature of the air flowing over the AFE is kept constant by actuating on the Peltier element current. Two large fans ensure the coupling of the inner and out volume to the Peltier and additional fans are monted on each ADC module to optimise the coupling of the airflow to the AFE. Passive heat exchangers are glued onto all ADCs and FPGAs.

independent cards.

The dense placement of the various components on the breadboard caused not only a strong heat dissipation, but also strong temperature fluctuations. An overview of the heat sources is given in Figure 11. To achieve the desired performance levels we therefore implemented an active thermal stabilisation of the whole system. To this end, we designed a double-walled housing and integrated a Peltier element into the top, which was coupled to both heat baths via additional fans. A diagram of the stabilisation is shown in Figure 12. Using a temperature sensor placed close to the AFE, we implemented an active temperature stabilisation which actuated on the current through the Peltier element. Figure 13 shows the thermal spectra, measured with temperature sensors positioned close to the AFE, on each ADC card with the system open in air and with active stabilisation. We were able to suppress the temperature fluctuations at the AFE to a level below $200 \mathrm{mK} / \sqrt{\mathrm{Hz}}$ at $1 \mathrm{mHz}$. 


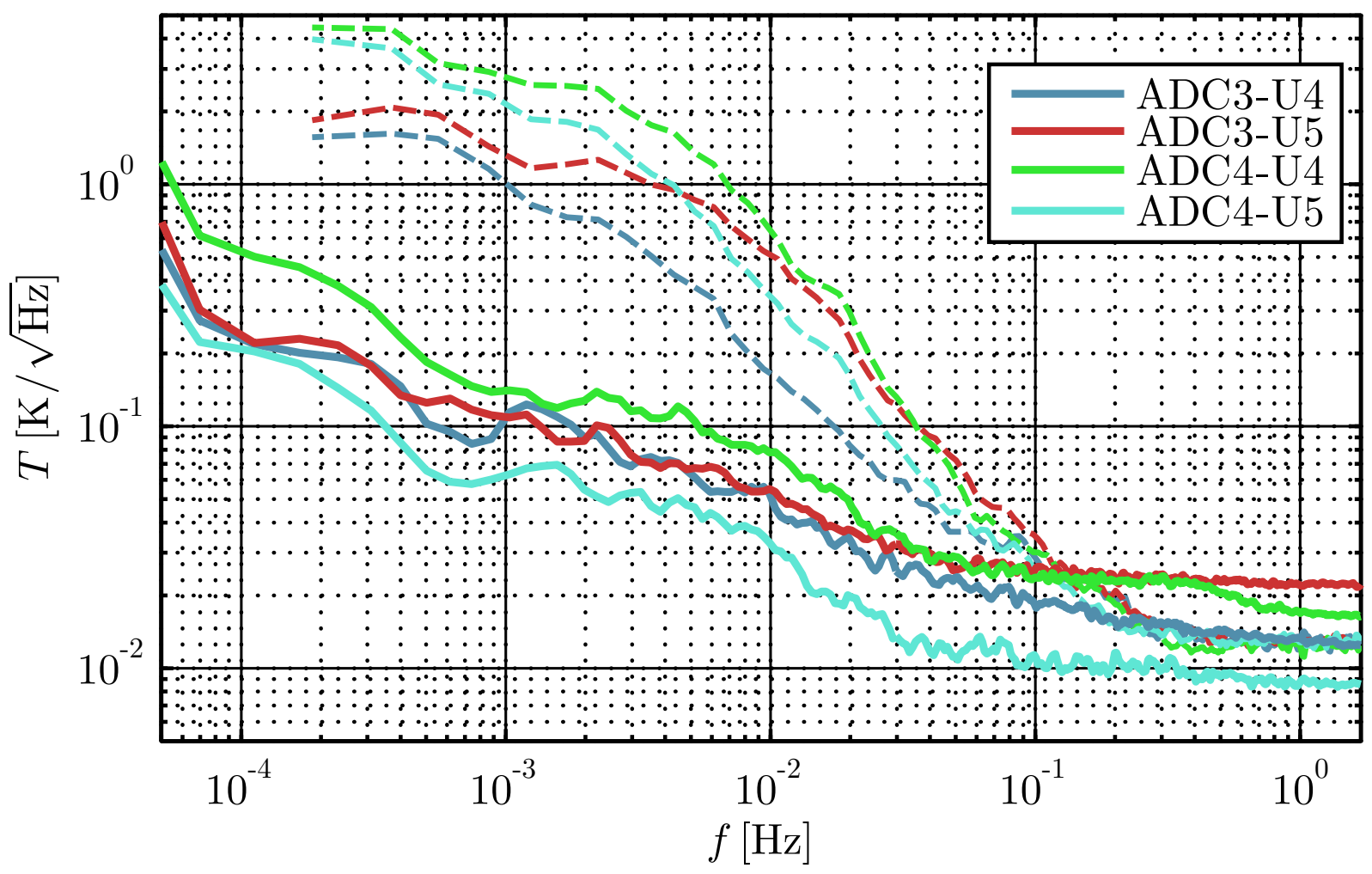

FIG. 13. Temperature spectral density measured with the sensors placed next to the AFE of the two ADC cards used during the measurement campaign. Shown are the values while operating the system in air (dashed lines), and while using the active temperature stabilisation in a dedicated housing (solid lines).

Using that thermal stabilisation we were able to achieve full performance for all frequencies with input white noise corresponding to a SNR of $75 \mathrm{dBHz}$ and $95 \mathrm{dBHz}$; as expected in intersatellite interferometers with long arms and low received optical power. Figure 14 shows the results of two measurements from our campaign. The first measurement (dashed lines) was done using a standard signal generator, locked to the phasemeter clock, to generate a clean input signal while operating the system in air. The performance is limited in all channels by some $1 /$ f noise (due to thermal noise) and no excess white noise is present in any of the channels. The second measurement (solid lines) was done with the full LISA-like input signal at $75 \mathrm{dBHz}$ SNR and with the active temperature stabilisation. For most channels we achieved the desired performance and all other measurements using higher SNR and lower signal frequencies showed even better performance. Similar to our earlier investigations with 


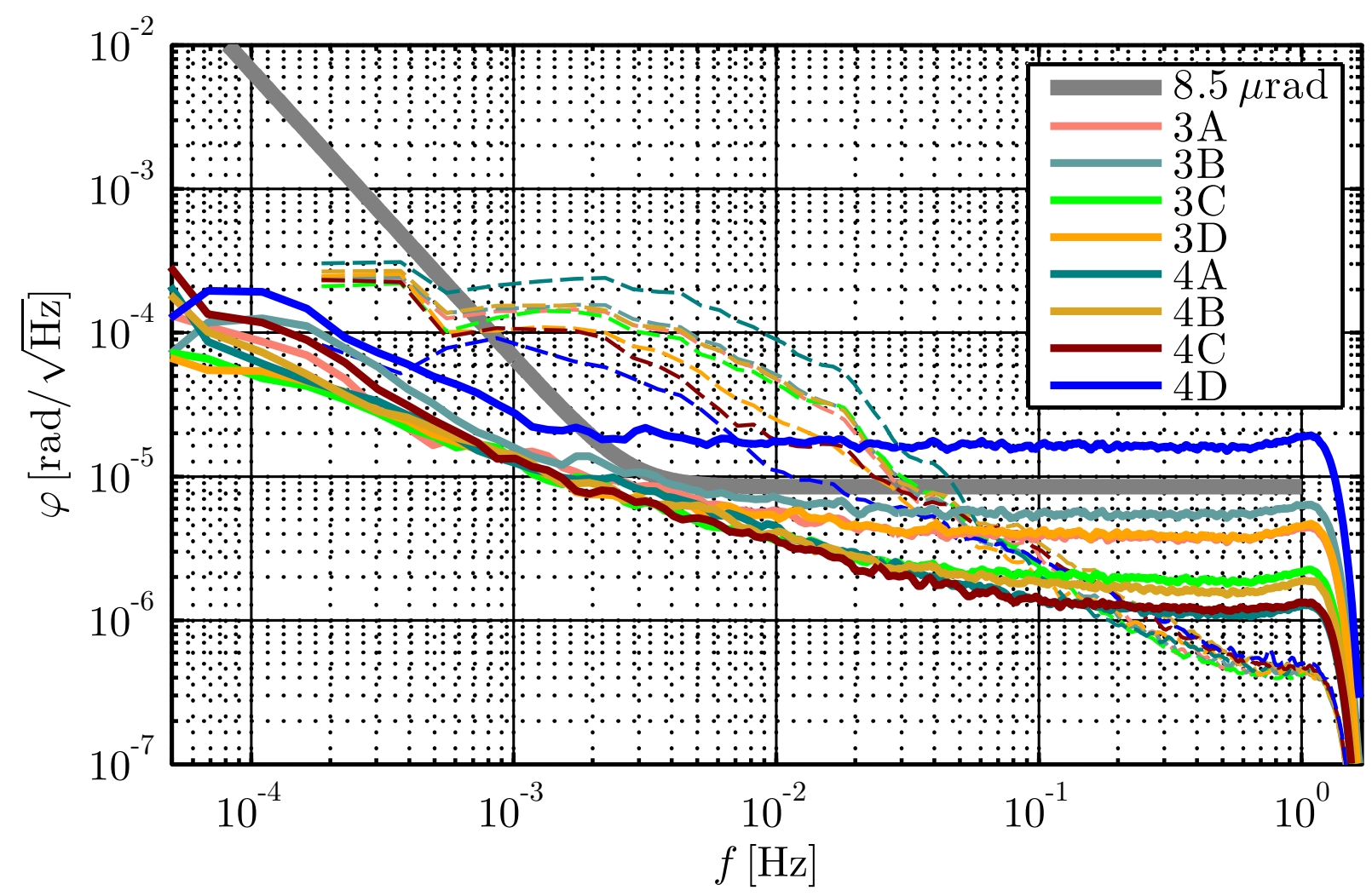

FIG. 14. Phase spectral densities for two measurements at $25 \mathrm{MHz}$ signal frequency, one with temperature stabilisation (solid lines) and one without (dashed lines).

the 16 channel prototype we find, again, a channel dependent white noise floor. From the difference to the first measurement we can directly conclude that this effect is driven by the signal dynamics, which were minimal in the first measurement, leading to no excess white noise. We investigated the cause for observing this coupling without different cable lengths only shortly. We found a dependency of this coupling on the resistor value matching in our resistive power splitter used to distribute the signal. Hence, we speculate that this noise is another example for a small vector noise that is related to the signal dynamics, which is either caused by impedance mismatches, cross talk, or a combination of these effects.

\section{CONCLUSION}

In this article we have presented a conceptual and experimental analysis of effects in the analogue measurement chain that can spoil the performance of phase readout systems at low frequencies. We have performed various experimental investigations with prototype readout 
systems and found a wide variety of set-ups that are suitable to achieve performance for certain frequencies. We were able to implement these prototypes with a mixture of commercially available hardware and additionally constructed analogue circuits for distributing and adding a pilot tone.

The design of a full LISA phasemeter prototype, able to operate over the whole desired frequency range, was found to be more demanding, requiring, amongst other things, an active temperature stabilisation of the analogue front-end. With the LISA phasemeter prototype we have demonstrated full phase readout performance of better than $\sqrt{2} \cdot 2 \pi \mu \mathrm{rad} / \sqrt{\mathrm{Hz}}$ for a single channel with LISA like input signals in null-measurement schemes.

Some of our measurements indicate the presence of non-linear noise couplings due to small vector signals. We have discussed the need to take such couplings into account and we described its influence in some detail. Due to the lack of sensitivity to many of these effects, we argue that other types of performance tests have to be implemented in the future, that take such influences into account. Three signal tests involving the whole measurement chain are, in our perspective, the preferred method to do that.

\section{ACKNOWLEDGMENTS}

We acknowledge funding by the European Space Agency (ESA) within the technology development project "LISA Metrology System" and "Optical Bench Development for LISA". The authors also gratefully acknowledge support by the International Max-Planck Research School for Gravitational Wave Astronomy (IMPRS-GW), by the Centre for Quantum Engineering and Space-Time Research (QUEST) and by Deutsches Zentrum für Luft- und Raumfahrt (DLR) with funding from the Bundesministerium für Wirtschaft und Technologie (project reference 50 OQ 0601).

\section{REFERENCES}

${ }^{1}$ K. Danzmann et al., "The Gravitational Universe: Whitepaper for the ESA L2/L3 selection," arXiv preprint arXiv:1305.5720 (2013).

${ }^{2}$ B. S. Sheard, G. Heinzel, K. Danzmann, D. A. Shaddock, W. M. Klipstein, and W. M. Folkner, Journal of Geodesy 86, 1083 (2012). 
${ }^{3}$ D. Shaddock, B. Ware, P. Halverson, R. E. Spero, and B. Klipstein, AIP Conf. Proc. 873, 689 (2006).

${ }^{4}$ O. Gerberding, B. Sheard, I. Bykov, J. Kullmann, J. J. E. Delgado, K. Danzmann, and G. Heinzel, Classical and Quantum Gravity 30, 235029 (2013).

${ }^{5}$ S. P. Francis, T. T.-Y. Lam, K. McKenzie, A. J. Sutton, R. L. Ward, D. E. McClelland, and D. A. Shaddock, Opt. Lett. 39, 5251 (2014).

${ }^{6}$ Y. Yu, S. Mitryk, and G. Mueller, Phys. Rev. D 90, 062005 (2014).

${ }^{7}$ H.-S. Liu, Y.-H. Dong, Y.-Q. Li, Z.-R. Luo, and G. Jin, Review of Scientific Instruments 85, 024503 (2014).

${ }^{8}$ Y.-R. Liang, H.-Z. Duan, X.-L. Xiao, B.-B. Wei, and H.-C. Yeh, Review of Scientific Instruments 86, 016106 (2015).

${ }^{9}$ O. Gerberding, S. Barke, I. Bykov, K. Danzmann, A. Enddaard, J. J. Esteban, A. Gianolio, T. V. Hansen, G. Heinzel, A. Hornstrup, O. Jennrich, J. Kullmann, S. M. Pedersen, T. Rasmussen, Z. Sodnik, and M. Suess, in ASP Conference Series; 9th LISA Symposium, ASP Conference Series, Vol. 467, edited by G. Auger, P. Binetruy, and E. Plagnol (2012) pp. $271-275$.

${ }^{10} \mathrm{~S}$. Barke, N. Brause, I. Bykov, J. J. Esteban Delgado, A. Enggaard, O. Gerberding, G. Heinzel, J. Kullmann, S. M. Pedersen, and T. Rasmussen, "LISA metrology systemfinal report," online: http://hdl.handle.net/11858/00-001M-0000-0023-E266-6 (2014).

${ }^{11}$ M. Tröbs, L. dArcio, S. Barke, J. Bogenstahl, I. Bykov, M. Dehne, C. Diekmann, E. Fitzsimons, R. Fleddermann, O. Gerberding, et al., in Proceedings of the 9th International Conference on Space Optics, Ajaccio (2012).

${ }^{12}$ R. Spero, B. Bachman, G. de Vine, J. Dickson, W. M. Klipstein, T. Ozawa, K. McKenzie, D. A. Shaddock, D. Robison, A. Sutton, and B. Ware, Class. Quantum Grav. 28, 094007 (2011).

${ }^{13}$ D. Schütze, G. Stede, V. Müller, O. Gerberding, T. Bandikova, B. S. Sheard, G. Heinzel, and K. Danzmann, Opt. Express 22, 24117 (2014).

${ }^{14}$ F. G. Cervantes, J. Livas, R. Silverberg, E. Buchanan, and R. Stebbins, Classical and Quantum Gravity 28, 094010 (2011).

${ }^{15}$ R. A. Wannamaker, The theory of dithered quantization. (University of Waterloo, 2003).

${ }^{16}$ B. Carter, Op Amps for everyone (Elsevier, 2003).

${ }^{17}$ R. Boudot and E. Rubiola, Ultrasonics, Ferroelectrics and Frequency Control, IEEE Trans- 
actions on 59, 2613 (2012).

${ }^{18}$ G. Hechenblaikner, J. Opt. Soc. Am. A 30, 941 (2013).

${ }^{19}$ M. Tröbs, L. d'Arcio, S. Barke, J. Bogenstahl, C. Diekmann, E. Fitzsimons, O. Gerberding, J. Hennig, F. Hey, H. Hogenhuis, et al., in Astronomical Society of the Pacific Conference Series, Vol. 467 (2013) p. 233.

${ }^{20}$ A. Joshi, S. Datta, J. Rue, J. Livas, R. Silverberg, and F. Guzman Cervantes, in SPIE Astronomical Telescopes+ Instrumentation, Vol. 8453 (2012) pp. 84532G-84532G-10.

${ }^{21}$ DDSP, "FMC10x user manual," http://www.4dsp.com/FMC107.php (2011).

${ }^{22}$ 4DSP, "FMC116/FMC112 user manual," http://www.4dsp.com/FMC116.php (2011).

${ }^{23}$ G. Heinzel, J. J. Esteban, S. Barke, M. Otto, Y. Wang, A. F. Garcia Marin, and K. Danzmann, Classical and Quantum Gravity 28, 094008 (2011).

${ }^{24}$ J. J. E. Delgado, Laser ranging and data communication for the laser interferometer space antenna, Ph.D. thesis, Leibniz Universität Hannover (2012).

${ }^{25}$ A. J. Sutton, K. McKenzie, B. Ware, G. de Vine, R. E. Spero, W. Klipstein, and D. A. Shaddock, Class. Quantum Grav. 30, 075008 (2013). 